\title{
A Game Theoretic Approach to Age of Information in Modern Random Access Systems
}

\author{
Leonardo Badia \\ Dept. of Information Engineering \\ University of Padova, Italy \\ email: leonardo.badia@unipd.it
}

\author{
Andrea Munari \\ Institute of Communications and Navigation \\ German Aerospace Center (DLR), Weßling, Germany \\ email: andrea.munari@dlr.de
}

\begin{abstract}
The age of information is a performance metric describing the freshness of updates, and is of particular interest for remote sensing scenarios involving high number of nodes, as is expected to happen, e.g., in emergency scenarios or industrial applications of the Internet of Things. Only recently, this metric received a rigorous treatment in the context of modern random access techniques that are realistic for this kind of systems. In this paper, we extend these investigations from the perspective of game theory, to discuss the role of individual strategic choices on the resulting system performance. We highlight how the introduction and fine-tuning of some parameters, specifically a cost for transmission from the nodes, can regulate the overall performance and achieve system-wide efficient allocations that are also equilibria for the selfish needs of the individual users.

Index Terms-Age of Information; Game theory; Irregular repetition slotted ALOHA; Internet of Things.
\end{abstract}

\section{INTRODUCTION}

In many communication systems involving remote sensing and distributed monitoring, it is important to assess the freshness of information that the system can gain through sporadic updates. This is captured by the age of information (AoI) [1], which is gaining increasing prominence in many analytical frameworks besides standard performance indicators such as throughput or delay.

Especially, AoI can be more relevant than these traditional metrics whenever the sensing nodes act without centralized coordination to send a limited amount of data, often through short packets, which are required to be kept up-to-date more than being transmitted in heaps. We argue that many scenarios in the industrial Internet of Things (IoT), such as remote estimation or mission-critical communications [2], and most of the commercial solutions and standards for IoT systems relate to this kind of applications [3], [4]. We also remark that in these contexts, random-based multiple access techniques are commonly used, which essentially amount to variations of the well-known ALOHA protocol [5].

Indeed, such scenarios are characterized by a high number of nodes, which are often heterogeneous in nature and have severe constraints in terms of power and computational capabilities. This would make their centralized control impractical, and justifies the need for distributed approaches, often built on random access techniques [6]. Moreover, an appealing theoretical tool that is often proposed in these cases, for both purposes of characterizing the system performance and even devising practical solutions is that of game theory, i.e., the study of multi-agent multi-objective decision problems [7], [8]. This implies that nodes are assumed to follow a selfish and sometimes opportunistic behavior, since they only care about their individual goals and can exploit particular circumstances taking place in the system to their own advantage [9]. Despite these objectives being sometimes at odds with the purpose of achieving a global system-wide efficiency, much more practical solutions can be obtained, toward which nodes are incentivized as they reflect their own goals.

It can be argued that the rationale behind random access techniques is related to this characteristic of game theory, which is also confirmed by the development of many game theoretic investigations of their performance [10]-[14]. However, virtually all of these investigations consider the individual player objectives as solely related to throughput, thereby ignoring the extensions to scenarios where AoI is more relevant.

At the same time, previous recent contributions discussed and gave precise evaluations of AoI in the context of a large number of nodes accessing a shared channel to maintain up-to-date information at their destinations [15]-[18]. However, most of the theoretical investigations usually consider either scheduling for collision-free transmissions or classic random-based medium access control protocols such as slotted ALOHA; only very recently in [19] modern access techniques more appropriate for large-scale IoT systems such as irregular repetition slotted ALOHA (IRSA) [20] were examined.

The goal of the present contribution is to combine all of these aspects in a novel way, to derive a game theoretic analysis of multiple nodes contending for access following modern random access, most specifically IRSA, and primarily aiming at minimizing the AoI of their data. The actual individual objective of the nodes is set as a linear combination of the AoI and a transmission cost, which is shown to be necessary to mitigate aggressive transmissions that would happen in a cost-free scenario. On the other hand, this can also be seen as a parameter that is further tuned by the system management to achieve an efficient operating point for the network.

The rest of this paper is organized as follows. In Section II, we review related contributions, also highlighting how our present contribution fills a gap in the recent literature. In Section III, we discuss, based on previous existing results, the foundations for our game theoretic analysis of IRSA, which is 
further developed in Section IV. Numerical results are shown in Section V and finally Section VI concludes the paper.

\section{RELATED WORK}

Many papers in the recent literature focus on the AoI metric, especially using it to capture the performance of remote sensing in IoT systems. This is also reminiscent of investigations of service availability or survivability in mission-critical application [21]. Already the seminal paper [1] considers slotted ALOHA as one of the reference scenarios; IRSA [20] can be considered as a generalization of this technique. Other recent investigations have characterized AoI in ALOHA-like systems and how to improve that metric [22], [23].

A detailed analysis of AoI for IRSA systems is presented in [19]. The derivations of that paper put the AoI in close relationship with the system throughput, so that performance metrics with different meanings are nevertheless found to be interconnected. Hence, our present contributions also relates to those papers discussing the throughput performance of IRSA, such as [24] where the protocol is characterized for low system loads, and [25] doing so for high loads instead.

There are also papers exploiting game theory to analyze the distributed exchange of data in IoT systems. Their approaches mostly relate to explicitly adversarial setups (for issues such as protection against denial-of-service, jamming, or other security aspects [9], [14]), which explains their resorting to game theory. In case of medium access evaluations, there are classic references considering ALOHA-like access protocols, such as [11]-[13], but they mostly revolve around the objectives of the players being to maximize their throughput. One general insight that can be gained is that selfish players tend to get inefficient equilibria in terms of throughput as opposed to globally optimal operating points [10]. One can expect this condition to extend to AoI, since for both metrics collisions must be kept to a minimum to get an efficient operating point. Moreover, the description of the medium access in these papers is often a generic characterization where collisions are to be avoided, and they do not consider modern random based access procedures such as IRSA.

Given the relatively recent spread of papers investigating AoI as the key metric [1], there are relatively fewer papers concerning games of AoI minimization; we can mention [18] where a ultra-dense IoT system is analyzed through mean-field games. Here, a generic carrier-sense multiple access is considered, for which the nodes switch through idle, waiting, or transmitting states. Also [26] considers nodes competing over a shared resource to get low AoI, but the access protocol is once again modeled as a plain collision-based model. Finally, [27] considers instead a game between two transmitter/receiver pairs but the shared medium is an interference channel, so the analysis refers to the physical layer and the achievable capacity, and not the medium access control.

\section{ReVISITING IRSA through GAME TheORY}

In this section, we summarize the most important findings of [19], and we explain how those conclusions can be extended to a game theoretic framework, which is the main focus of the present contribution. Throughout our discussion, we assume a population of $n$ terminals that share a common channel. Time is divided in slots of equal duration, tuned to allow transmission of a data packet, and nodes are assumed to be synchronized to such pattern. At each slot, terminal $i$ becomes active with probability $\rho_{i}$ (referred to as activation or transmission probability), generating a time-stamped update addressed to a common sink. Without loss of generality, nodes will be modeled as a single-packet buffers, so that at any time each device either has the latest generated packet to transmit, or does not have data to send.

Access to the medium is regulated by IRSA. Accordingly, time is organized into frames of $m$ slots each, and the first transmission opportunity for a newly generated packet is at the start of the successive frame. Each user with data to send accesses the channel by sending $\ell$ replicas of its data unit, uniformly placed at random across the slots of a frame. The number of copies is drawn from a probability distribution $\left\{\Lambda_{\ell}\right\}$, common to all terminals. At the receiver side, decoding starts once the whole frame has been retrieved, and relies on successive interference cancellation. In particular, assuming a simple collision channel model, a packet sent over a collisionfree slot can be decoded, and the interference contribution of its twin replicas can be removed from the corresponding slots, possibly leading to other singleton and thus decodeable time units. The procedure continues until either all users have been decoded, or no more singleton slots are available over the frame. For a more detailed description of the protocol, the interested reader is referred to [20]. We note that, for the system model under consideration, a node accesses the channel at the start of a frame if it has become active at least once over the previous $m$ slots, i.e., with probability $1-(1-\rho)^{m}$. Accordingly, the channel load $\mathrm{G}$, defined as the average number of users accessing the channel per slot is

$$
\mathbf{G}=\frac{n\left(1-(1-\rho)^{m}\right)}{m} .
$$

In this setting, we are interested in the average AoI for a node, defined as [1]

$$
\Delta:=\lim _{T \rightarrow \infty} \frac{1}{T} \int_{0}^{T}(t-\sigma(t)) d t
$$

where $\sigma(t)$ denotes the time stamp of the last update successfully received at the sink for the node of interest. In [19], it was shown that the this quantity can be computed in closed-form when IRSA is employed, and the overall formula contains terms that can be relevant in a game theoretic analysis, the most important of which relates to the total throughput of the system, denoted as S. Specifically, the main finding can be summarized stating that the average AoI for an IRSA system with $n$ users and operating over frames of size $m$ slots reduces to [19, Eq. (21)]

$$
\Delta_{\text {irsa }} \simeq \frac{1}{2}+\frac{n}{\mathrm{~S}}+m .
$$



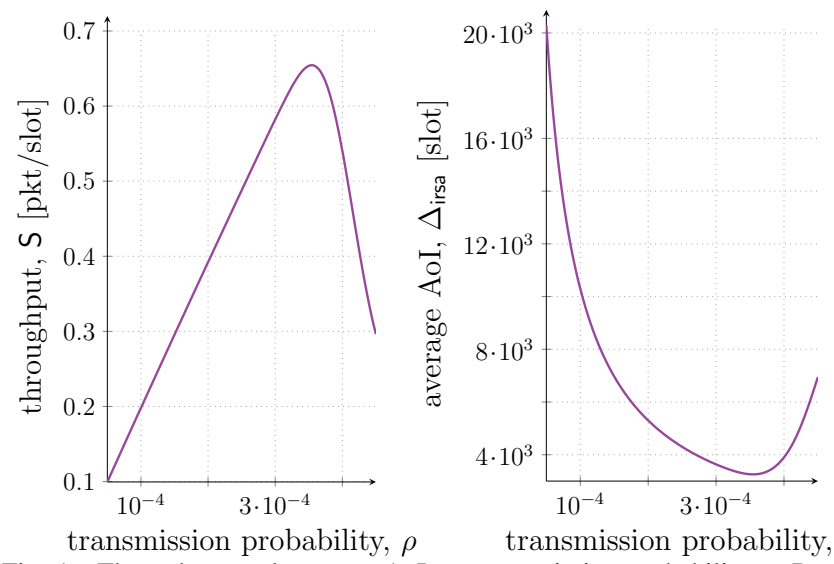

Fig. 1. Throughput and average AoI vs. transmission probability $\rho$. Result derived following the analytical approximations in [19]. In both cases, $n=$ 2000, $m=200$ [slots].

Eq. (3) is a very tight approximation in practical IoT settings, characterized by large values of $n$ and sufficiently low transmission probability $\rho$ of each individual user, and is especially useful for the present discussion. We note, however, that an exact closed-form expression is also available for any set of parameters [19] and could be employed without altering our main conclusions. In practice, the consequence of (3) is that the individual choices of the users only affect the throughput, and in turn reflect on the AoI. In this spirit, the main insight is that the more often the random access users succeed in their transmission without colliding, the higher the throughput and similarly the lower the AoI. Such a conclusion generalizes the findings already known for simpler random access protocols such as slotted ALOHA [1].

While an exact characterization of $S$ is still elusive, a good analytical approximation can be obtained combining two different results [24], [25], which provide the packet success probability $P_{\text {succ }}$ of IRSA for low and high loads, respectively. Such operation regimes are also called the error floor and the waterfall regions. Leaning on these expressions, the throughput can be easily evaluated as $S=G \cdot P_{\text {succ }}(G)$, where the success probability follows a different trend depending on whether the IRSA system operates in the error floor or the waterfall regime [19]. Ultimately, this implies that the throughput relates to the combined choices of all the nodes, and more specifically on their decision about whether to transmit or not, represented by the transmission probability $\rho$. In particular, while $\mathrm{G}$ is rapidly increasing in $\rho$ (see (1)), $P_{\text {succ }}$ is not, which leads to the conclusion that the value of $\rho$ maximizing $\mathrm{S}$ is between 0 and $1{ }^{1}$ For reference, the trends obtained for throughput and average AoI following the presented approach are reported in Fig. 1 for the case of $n=2000$ terminals and $m=200$ slots. For additional insights, please refer to [19].

In the present paper, we give a different twist to these derivations by highlighting that the aforementioned scenario

\footnotetext{
${ }^{1}$ Incidentally, we note that, for the specific case of a slotted ALOHA obtained for $m=1$, it is well known that such a value is $\rho=1 / n$.
}

describes a quintessential game theoretic situation, where individual combined choices, collected together, result in a global system outcome that is nevertheless perceived by the players as consistent with their own benefit. In other words, we just need to highlight the individual choices of one single user, as opposed to the others. In particular, we focus on a specific node $i$ of the IRSA system, and we consider its transmission probability $\rho_{i}$ as its individual choice. Remarkably, we can characterize the transmission probability of all other nodes $j \neq i$ with a similar approach, but symmetry considerations immediately lead us to conclude that every reasonable system outcome will have the same transmission probability for any other node, hence we denote it as $\rho_{-i}{ }^{2}$ Quite logically, the same reasonings also imply that also $\rho_{i}=\rho_{-i}$ must hold, at least if an equilibrium condition is reached. Yet, we keep these notations separate as denoting the actions of different players.

Consequently, (1) can be rewritten as

$$
\mathrm{G}\left(\rho_{i}, \rho_{-i}\right)=\frac{1-\left(1-\rho_{i}\right)^{m}+(n-1)\left(1-\left(1-\rho_{-i}\right)^{m}\right)}{m}
$$

where we split the action of player $i$ from those of the other players. Thus, the system throughput can be derived as

$$
\mathrm{S}\left(\rho_{i}, \rho_{-i}\right)=\mathrm{P}_{\text {succ }}\left(\rho_{i}, \rho_{-i}\right) \mathrm{G}\left(\rho_{i}, \rho_{-i}\right)
$$

where $\mathrm{P}_{\text {succ }}\left(\rho_{i}, \rho_{-i}\right)$ depends on the operation regime and can be derived as per [19].

Finally, we assume that the individual node $i$ incurs a cost for transmission that is proportional to probability $\rho_{i}$ through a constant $c$ [10]. Since, according to the discussion above, the transmission probabilities are expected to be in the same order of magnitude as $1 / n$, it may also be convenient to denote $\tilde{c}=c / n$. As a result, we can define the utility of node $i$ as

$$
u_{i}\left(\rho_{i}, \rho_{-i}\right)=-\Delta_{\mathrm{irsa}}-c \rho_{i}=-\frac{n}{\mathrm{~S}\left(\rho_{i}, \rho_{-i}\right)}-c \rho_{i}+K
$$

where $K$ is a fixed term that is meant to collect all constants in (3); a similar equation can also be written for player $-i$. In game theory, utilities represent the individual objectives that players want to maximize, hence the reason for the negative sign in the above equation [8]. It is also worth mentioning that constant biases of the utilities such as the term $K=\frac{1}{2}+m$ do not influence the outcome as they give the same order of preferences for the players, the same equilibria, and so on. In the following, we will neglect the value of $K$, since even imposing $K=0$ in the definition of the utilities does not change what follows.

The reason for introducing the cost term is twofold. On the one hand, terminals may indeed be assumed to incur a cost, e.g., related to power consumption, for every transmission. Thus, the insertion of that term is sensible and having it in the utility function of each player is in line with similar studies [11], [12], [14]. On the other hand, the introduction of the cost is required from a mathematical standpoint to have the

\footnotetext{
${ }^{2}$ It is customary in game theory to use the notation "- $i$ " to denote the choices of all other players except for player $i$, so that the scenario is reduced to a 2-player game between $i$ and $-i$.
} 
system working on a desirable equilibrium. As will be shown in the following, if transmissions are cost-free, nodes have an incentive towards persistent transmission with $\rho=1$, which undermines the system efficiency; a cost term can limit such a behavior. Such a conclusion is also valid for the standard investigations of ALOHA-like systems where the utilities are related to the throughput achieved by the node, and not its AoI. Still, using AoI-based utilities as those in (6) has a different impact on the resulting equilibrium and the subsequent system performance, as detailed in the next section.

\section{GAME TheORETIC ANALYSis}

The setup considered in the previous section can be used to formulate an $n$-player static game of complete information among the nodes of the IRSA network. For the aforementioned symmetry reasons, we can consider each player $i$ to choose its own transmission probability $\rho_{i} \in[0,1]$ independently and without coordination with the others, with the goal of maximizing its own utility $u_{i}\left(\rho_{i}, \rho_{-i}\right)$ captured by (6). The game is considered to be static since fully rational players are immediately able to derive the consequences of their chosen $\rho_{i}$ and are also aware that the other nodes will do the same. The assumption of complete information, instead, corresponds to assuming that all nodes are aware of this setup and that the other nodes follow (6) as well.

In this context, we look for a possible Nash equilibrium (NE), which is generally the instrument of choice in game theory to identify the operating point of the system, as it describes a strategic choice of the players where no one has an incentive to deviate from [7]. Given that the game uses continuous valued strategies of the player in $[0,1]$ and also the utilities are continuous and once derivable in $\rho_{i}$, the NE can promptly found by imposing the condition

$$
\frac{\mathrm{d} u_{i}}{\mathrm{~d} \rho_{i}}=0 \quad \text { for every } i .
$$

Actually, since $\rho_{i}$ is chosen within the interval $[0,1]$, also the extreme values 0 and 1 should be checked for possible maxima, and their role will be discussed in what follows.

We remark that imposing (7) is actually quite different from the system-wide minimization of the AoI (or maximization of the throiughput) discussed in the previous section, which corresponds to finding the correct trade-off in the product $\mathrm{GP}_{\text {succ }}$ as depending on $\rho$, and where the two factors follow contrasting trends in $\rho$. In the game theoretic formalization, the maximization of the utilities (7) translates into

$$
\frac{\mathrm{dS}\left(\rho_{i}, \rho_{-i}\right)}{\mathrm{d} \rho_{i}} \cdot \frac{n}{\left(\mathrm{~S}\left(\rho_{i}, \rho_{-i}\right)\right)^{2}}=c .
$$

Moreover, it is reasonable to assume that, for large $n$, $\mathrm{P}_{\text {succ }}$ is unaffected by small variations of $\rho_{i}$ as long as $\rho_{-i}$ is kept constant. The reason is that the expressions for $P_{\text {succ }}$ in [19] mostly depend on the regime determined by the system-wide choice of the transmission probabilities of all the terminals, and a small variation of the individual behavior of one node does not significantly change that value. Hence, we can combine (5) and (8) to obtain

$$
\frac{\mathrm{dS}\left(\rho_{i}, \rho_{-i}\right)}{\mathrm{d} \rho_{i}} \approx \mathrm{P}_{\text {succ }}\left(\rho_{i}, \rho_{-i}\right)
$$

and the NE condition translates into

$$
\mathrm{S}\left(\rho_{i}, \rho_{-i}\right) \cdot \mathrm{G}\left(\rho_{i}, \rho_{-i}\right)=\frac{n}{c}=\tilde{c}^{-1} \quad \text { for every } i .
$$

Clearly, such a condition cannot be met if $c=0$. Thus, we conclude from (10) that a cost-free transmission implies the push for every node $i$ to always increase its transmission probability towards $\rho_{i}=1$. This conclusion is analogous to what can be found in other studies of random access games when considering utilities related to throughput [11], [12]. Indeed, whenever the transmission probabilities of all other players $\rho_{-i}$ are set, it is always convenient for player $i$ to unilaterally deviate and choose a value of $\rho_{i}$ higher than the others, since it will obtain a better throughput and a lower AoI. Eventually, symmetry considerations lead to the conclusion that the only $\mathrm{NE}$ is obtained when $\rho_{i}=\rho_{-i}=1$, where no player can get an advantage to deviate from. However, this is also a bad operating point for the system as it corresponds to a vanishing throughput or diverging AoI.

As discussed, this justifies the role of a cost term $c>0$ as both a sensible extension to account for practical aspects and a way to regulate the behavior of the individual user. One may argue whether the dimensioning of $c$ should relate to either of these aspects, and in general the numerical setup may not be immediate. Depending on the expression of $\mathrm{S}\left(\rho_{i}, \rho_{-i}\right) \mathrm{G}\left(\rho_{i}, \rho_{-i}\right)$, one can get a non-degenerate $\mathrm{NE}$ whenever this product equals $\tilde{c}$. Actually, there are always two intersection points, but only the left-most of them is found to be a maximum for the individual player utility, the other being a minimum and an unstable operating point, which is in line with classical investigations of ALOHA-like protocols [10].

We also highlight an important advantage of our analysis where the individual objectives of the players relate to minimizing the AoI, as opposed to the more standard throughput maximization. If their objectives were connected to throughput, the inefficiency of the NE would be stronger as this implies a NE condition where the first derivative of the throughput equals $c$, which reflects on the success probability, as per (9). Such a condition implies that the range of practical cost values is limited (it must be equal to a legitimate value for $P_{\text {succ }}$ ). Instead, (10) is easier to solve for a non-degenerate $\mathrm{NE}$; intuitively, this may be explained by the remark that a low AoI can be achieved with relatively fewer successful updates than what needed for a high throughput. Especially, if the network manager wants to set an AoI-optimal NE, it can simply compute the optimal throughput value $\mathrm{S}^{*}$ of the IRSA network minimizing the AoI, and its associated value of $\mathrm{G}^{*}$, and set a cost $\tilde{c}=\left(\mathrm{S}^{*} \mathrm{G}^{*}\right)^{-1}$.

This equilibrium condition (10) can be seen as caused by the balance between higher throughput and the increase in the individual cost paid. While the latter is always constantly equal to $c$, the former saturates as $\rho_{i}$ grows, and the $\mathrm{NE}$ is reached 


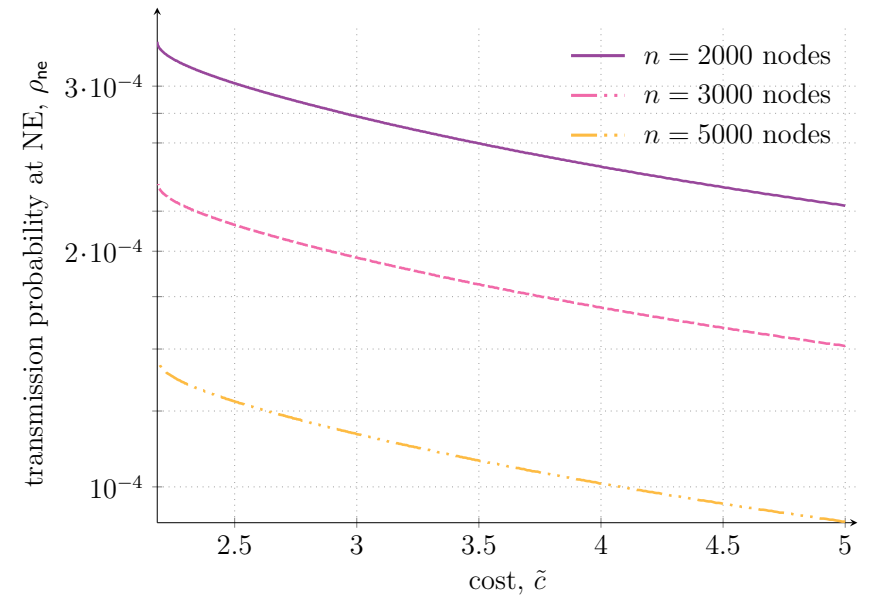

Fig. 2. Transmission probability $\rho_{i}$ at the NE vs. normalized cost $\tilde{c}$. Different lines denote different values of $n$. In all cases, $m=200$ [slots].

at their meeting point. A similar reasoning holds true from the perspective of global efficiency, where if we gradually increase the transmission probability for all nodes jointly, the best operating point is found when the incremental system benefit equals $c$; yet, the NE differs from a network-wide optimization in that a higher usage harms the individual users as $1 / n$ of what happens if the whole system is considered, see also (4). As a result, even when the cost term is effective in limiting the usage, the NE still corresponds to a higher transmission probability than what would get the optimal sum utility, i.e., a suboptimal global performance. This principle, known in the economic literature as the tragedy of the commons [28], that users driven by selfish interests tend to overuse the resource, is by no means exclusive to wireless networks and has applications in many game theoretic contexts.

\section{NumERICAL EVAluations}

To illustrate some of the key trends highlighted in Sec. IV, we focus on an IoT-inspired setup in which a large number $n$ of terminals contend for the same channel relying on IRSA. In all cases, the protocol is operated over frames of duration $m=200$ [slots], and a regular degree distribution $\Lambda(x)=x^{3}$ is employed, i.e., each active terminal transmits three copies of its packet uniformly distributed over the frame. We regard a study of the impact of different distributions as well as of different frame sizes as relevant part of our future work.

A first insight is offered by Fig. 2, which reports the transmission probability leading to a NE when varying the normalized cost $\tilde{c}$. In the plot, different lines denote the results obtained for different user populations. As pointed out in Sec. IV, too low costs lead to equilibria characterized by $\rho_{i}=\rho_{-i}=1$, which are of no practical interest. We therefore restrict our analysis to values of $\tilde{c}$ which intersect the curve $\mathrm{S}\left(\rho_{i}, \rho_{-i}\right) \mathrm{G}\left(\rho_{i}, \rho_{-i}\right)$ and offer non-degenerate NEs. As expected, larger costs induce nodes to scatter the activation pattern due to the higher penalty undergone with each transmission. The effect becomes more pronounced as the network population increases, as the more intense contention requires

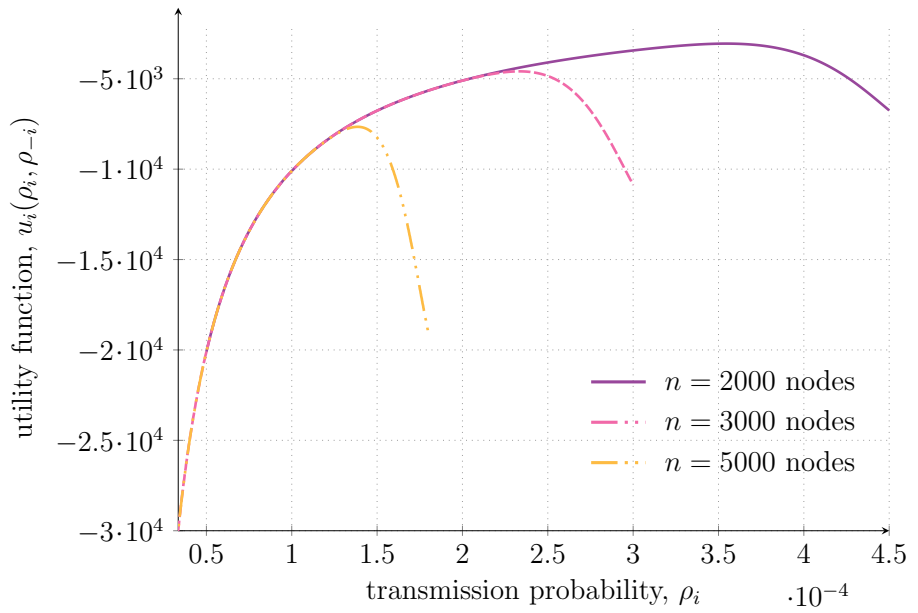

Fig. 3. Utility for user $i, u_{i}\left(\rho_{i}, \rho_{-i}\right)$, vs. transmission probability $\rho_{i}$. Different lines denote different values of $n$. In all cases, $m=200$ [slots].

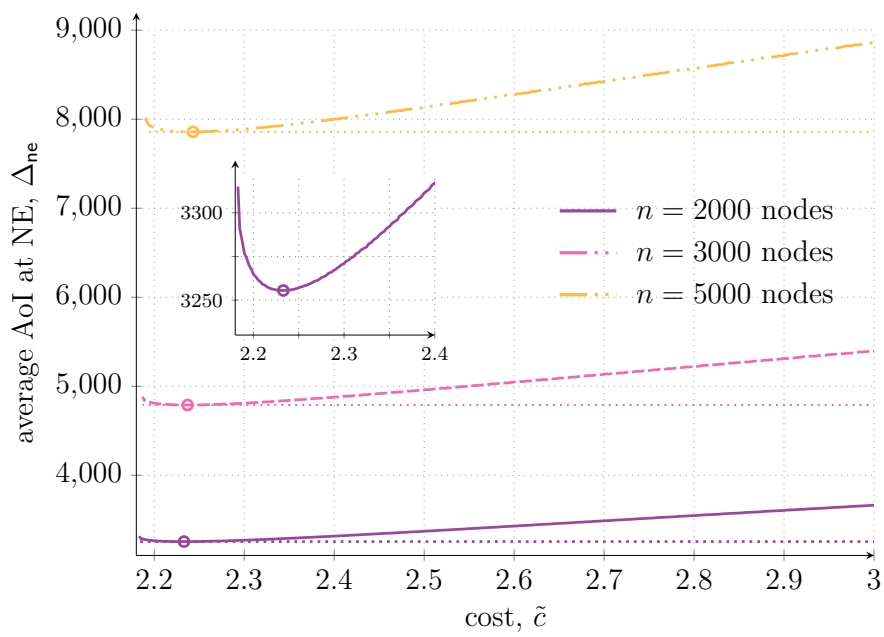

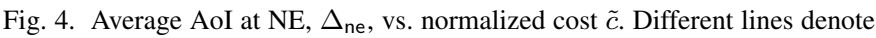
different values of $n$. In all cases, $m=200$ [slots].

terminals to access the channel less frequently to maintain reasonable throughput levels.

The utility function achieved by a terminal is in turn shown in Fig. $3 .^{3}$ In line with (6), the trend is dominated by the average AoI, i.e., the term $-\Delta_{\text {irsa }}$. Accordingly, sporadic transmissions results in very low utilities, due to a too low update rate. When terminals become aggressive and attempt frequent delivery of status reports, channel congestion and the role of collisions start to emerge. The resulting decay in throughput and delivery rate drives the plummeting behavior exhibited by the utility curves in the rightmost region of the figure. Interestingly, the plot reveals the existence of a nontrivial operating point where users can maximize their utility.

The fundamental question on whether this equilibrium can be reached enjoying conditions that are optimal also from a network standpoint is tackled in Fig. 4, where the average AoI at the NE is reported against the normalized cost $\tilde{c}$.

\footnotetext{
${ }^{3}$ Without loss of generality, the reported results were obtained setting a normalized cost that lead to a NE which is optimum from an AoI networkwide perspective, see the discussion of Fig.4.
} 
The horizontal lines denote the minimum average AoI that can be obtained when the system operator can fully control the transmission probability $\rho$ of all the users, showing the ideal working point for the system, i.e., the minimum of (3). Remarkably, regardless of the number of terminals contending for the channel, a proper choice of $\tilde{c}$ can lead selfish users to operate in the desired way. It is also interesting that the optimal value of $\tilde{c}$ (circle markers) does not change significantly with $n$, suggesting a linear growth of the target cost with the number of users in the system. Beyond the specific numerical values, the presented trends pinpoint the importance and potential of tailored pricing policies [29], for which our analysis offers a preliminary yet useful design tool. This remark is further buttressed when observing that the achieved network-wide performance is rather sensitive to the value of $\tilde{c}$. This is better highlighted in the subplot reported within Fig. 4, which zooms in on the behavior of the average AoI for $n=2000$ in the proximity of its minimum, and reveals a stark increase of the metric when $\tilde{c}$ is reduced. This effect stems from the fact that the optimal AoI is reached for a NE that pushes the system close to its peak throughput performance. In such conditions, users respond to small reductions of the cost with an increase in the transmission probability that has dire consequences, leading to the well-known plummet in throughput and delivery ratio exhibited by IRSA [20], see Fig. 1.

\section{CONCLusions}

We presented a game theoretic analysis of modern random access for the Internet of Things, considering a network where a high number of nodes communicate following the IRSA protocol, with an individual objective related to minimizing their AoI and also their transmission cost. Based on previous analytical formulations of the AoI for IRSA system, we showed that our framework is able to set an AoI-efficient working point, doing so in a distributed fashion where nodes act without coordination and driven by selfish objectives. In practice, this translates the system-wide optimization to a more practical approach based on individual actions of each nodes.

Future work may consider an expanded game theoretic formulation where the strategic choices of the nodes are more complex than just setting their transmission probability, possibly considering some sort of feedback from the receiver and an overall planning ahead over multiple update epochs. Even for these scenarios, game theory can be the proper instrument to set a self-enforcing distributed management of nodes with minimal supervision from the network manager, which appear to be a desirable choice for future IoT implementations.

\section{REFERENCES}

[1] R. D. Yates and S. K. Kaul, "Status updates over unreliable multiaccess channels," in Proc. IEEE ISIT, 2017, pp. 331-335.

[2] T. Do-Duy, L. D. Nguyen, T. Q. Duong, S. Khosravirad, and H. Claussen, "Joint optimisation of real-time deployment and resource allocation for uav-aided disaster emergency communications," IEEE $J$. Sel. Areas Commun., 2021.

[3] LoRa Alliance Tech. Committee, "LoRaWAN 1.1 specification," Oct. 2017.
[4] J. Chen, K. Hu, Q. Wang, Y. Sun, Z. Shi, and S. He, "Narrowband internet of things: Implementations and applications," IEEE Internet Things J., vol. 4, no. 6, pp. 2309-2314, 2017.

[5] N. Abramson, "The ALOHA system: Another alternative for computer communications," in Proc. Fall Joint Comp. Conf., 1970, pp. 281-285.

[6] I. Leyva-Mayorga, C. Stefanovic, P. Popovski, V. Pla, and J. MartinezBauset, "Random access for machine-type communications," Wiley $5 G$ Ref: The Essential 5G Reference Online, pp. 1-21, 2019.

[7] A. B. MacKenzie and L. A. DaSilva, "Game theory for wireless engineers," Synth. Lect. Commun., vol. 1, no. 1, pp. 1-86, 2006.

[8] G. Quer, F. Librino, L. Canzian, L. Badia, and M. Zorzi, "Internetwork cooperation exploiting game theory and bayesian networks," IEEE Trans. Commun., vol. 61, no. 10, pp. 4310-4321, 2013.

[9] F. Jameel, S. Wyne, G. Kaddoum, and T. Q. Duong, "A comprehensive survey on cooperative relaying and jamming strategies for physical layer security," IEEE Commun. Surveys Tuts., vol. 21, no. 3, pp. 2734-2771, 2018.

[10] A. MacKenzie and S. Wicker, "Selfish users in ALOHA: a gametheoretic approach," in Proc. IEEE VTC Fall, vol. 3, 2001, pp. 13541357.

[11] L. Chen, T. Cui, S. H. Low, and J. C. Doyle, "A game-theoretic model for medium access control," in Proc. WICON, 2007.

[12] G. Tan and J. Guttag, "The 802.11 MAC protocol leads to inefficient equilibria," in Proc. IEEE Infocom, vol. 1, 2005, pp. 1-11.

[13] Y. Cho and F. A. Tobagi, "Cooperative and non-cooperative ALOHA games with channel capture," in Proc. IEEE Globecom, 2008, pp. 1-6.

[14] L. Badia and F. Gringoli, "A game of one/two strategic friendly jammers versus a malicious strategic node," IEEE Netw. Lett., vol. 1, no. 1, pp. 6-9, 2019.

[15] P. D. Mankar, M. A. Abd-Elmagid, and H. S. Dhillon, "Spatial distribution of the mean peak age of information in wireless networks," IEEE Trans. Wireless Commun., 2021.

[16] H. Tang, J. Wang, L. Song, and J. Song, "Minimizing age of information with power constraints: Multi-user opportunistic scheduling in multistate time-varying channels," IEEE J. Sel. Areas Commun., vol. 38, no. 5, pp. 854-868, 2020.

[17] O. T. Yavascan and E. Uysal, "Analysis of slotted ALOHA with an age threshold," IEEE J. Sel. Areas Commun., vol. 39, no. 5, pp. 1456-1470, 2021.

[18] B. Zhou and W. Saad, "Age of information in ultra-dense IoT systems: Performance and mean-field game analysis," arXiv preprint arXiv:2006.15756, 2020.

[19] A. Munari, "Modern random access: an age of information perspective on irregular repetition slotted ALOHA," IEEE Trans. Commun., vol. 69, no. 6 , pp. 3572-3585, 2021.

[20] G. Liva, "Graph-based analysis and optimization of contention resolution diversity slotted ALOHA," IEEE Trans. Commun., vol. 59, no. 2, pp. 477-487, 2010.

[21] S. Samarakoon, M. Bennis, W. Saad, and M. Debbah, "Predictive ultra-reliable communication: A survival analysis perspective," IEEE Communications Letters, vol. 25, no. 4, pp. 1221-1225, 2020.

[22] D. C. Atabay, E. Uysal, and O. Kaya, "Improving age of information in random access channels," in Proc. IEEE Infocom Wkshps, 2020, pp. 912-917.

[23] H. H. Yang, A. Arafa, T. Q. Quek, and H. V. Poor, "Age of information in random access networks: A spatiotemporal study," in Proc. IEEE Globecom, 2020, pp. 1-6.

[24] E. Sandgren, A. G. i Amat, and F. Brännström, "On frame asynchronous coded slotted ALOHA: Asymptotic, finite length, and delay analysis," IEEE Trans. Commun., vol. 65, no. 2, pp. 691-704, 2016.

[25] A. Graell i Amat and G. Liva, "Finite-length analysis of irregular repetition slotted ALOHA in the waterfall region," IEEE Commun. Lett., vol. 22, no. 5, pp. 886-889, 2018.

[26] K. Saurav and R. Vaze, "Game of ages," in Proc. IEEE Infocom Wkshps, 2020, pp. 942-947.

[27] G. D. Nguyen, S. Kompella, C. Kam, J. E. Wieselthier, and A. Ephremides, "Information freshness over an interference channel: A game theoretic view," in Proc. IEEE Infocom, 2018, pp. 908-916.

[28] G. Hardin, "The tragedy of the commons," J. Nat. Resource Pol. Res., vol. 1, no. 3, pp. 243-253, 2009.

[29] L. Badia, S. Merlin, A. Zanella, and M. Zorzi, "Pricing VoWLAN services through a micro-economic framework," IEEE Wireless Commun., vol. 13, no. 1, pp. 6-13, 2006. 